\title{
DEVELOPMENT OF A WHITE-CANE-EXTENSION TO Detect Automatic Doors
}

\author{
Wataru Uemura and Takato Hayama \\ Department of Electronics and Informatics, Ryukoku University, Shiga, Japan
}

\begin{abstract}
The majority visually impaired persons are using a white cane to move within their environment. They avoid to collision the obstacles by detecting objects when swinging white cane touches them. An automatic door is opened when it detects the person comes to it by the sensor, for example, infrared sensor. This kind of automatic doors are very useful because we need not touch the doors in order to open them. However, for majority visually impaired persons, it is difficult to detected automatic doors because they detect the object by touching. In this paper, we propose the detection method which tells the existence of automatic doors to the visually impaired person. The device is consisted of an infrared sensor and a vibration motor. The infrared sensor is put on the tip of the white cane and the vibration motor is put on close to the hand. And when it detects the infrared signal, the vibration motor runs. Then the visually impaired persons can through the automatic door in safety without any cares.
\end{abstract}

\section{KEYWORDS}

Visible impaired person, White-cane, infrared sensor

\section{INTRODUCTION}

Recently, the doors become automatically, then we need not to stop to open the doors. In Japan, the law about the barrier free was changed in 2006 and the guideline for the train system were shown. Then research reports about the automatic door at the station were published [1-3]. These reports proposed the improvement of the door sensors in order to decrease misdetections and the number of door movement became one-third than the conventional automatic door. On the other hand, for the visually impaired person it is difficult to find such automatic door because they need to touch it in order to detect it.

It is said that the information to get from the visual is obtained 83 percent for the human. Visually impaired person supplements its 83\%-information primarily with hearing, touching and their experience. They walk with the white cane which tells around information to themselves. Some difficulties are reported at [4] because walking style is different from normal. However, there is no useful and convenient tool instead of the white cane. So, we focus on the white cane.

The visually impaired person cannot find the location of the automatic door which needs not to be touched. Then their body and face are hit by the wall because they cannot recognize the centre of the automatic door and the border between the door and the wall. And when they check the door location by the white cane, sometimes the body hits to the door because only white cane goes through to the door. If they walk enter to the detection area of the automatic door using the only wall information by the white cane, there are dangerous, for example, the door moves quickly and hits them or enter their finger. The automatics door detects the person movement in order to open and close, using the sensors, in particular the infrared sensor. This paper proposes 
the detection method for the visually impaired person, which has the infrared sensor and the vibration motor. And the purpose of this system is to tell the existence of automatic doors to the visually impaired person. We put infrared sensor on the tip of the white cane and the vibration motor on close to the hand. And when it detects the infrared signal, the vibration motor runs. The sense in order to communicate with the visually impaired person must be not sense of sight but the other sensory organs. For example, in order to play the action game for the visually impaired person, [5] proposed using the notification sound as the user interface. In this paper, we assume outdoor usage, so we focus on the vibration as the notification method. So, the vibration motors are used for our proposed system. And the visually impaired persons can through the automatic door in safety without any cares.

Section 2 describes the mechanism of the automatic door, and we propose the detection device for the automatic door in Section 3. At Section 4, we have the experiment in order to show the performance by the proposed device. Finally, we conclude this paper in Section 5.

\section{About the Automatic Door AND ITS Sensors}

The automatic door is the device which moves the door to open and close by not the human power but the electronic power. One kind of the automatic door opens the door by the open button, the other opens the door by detection the person movement using the sensor. This paper focuses the latter.

\subsection{The Sensors of the Automatic Door}

The doors are opened by the motor which is controlled when the sensor detects the movement, like as the person and dynamic objects, and so on. There are about three kinds of sensors: 1) near-infrared spectroscopy, 2) far-infrared spectroscopy, and 3) microwave. In Japan, most of automatic door sensor are near-infrared spectroscopy. The near-infrared reflection sensor uses a near-infrared reflection detection method in which near-infrared rays are radiated in a certain range and the change in the reflection of the light is detected (shown in Figure. 1). So, most of Japanese automatic doors always emits the infrared rays. Then, we can detect the automatic door by detecting the infrared rays.

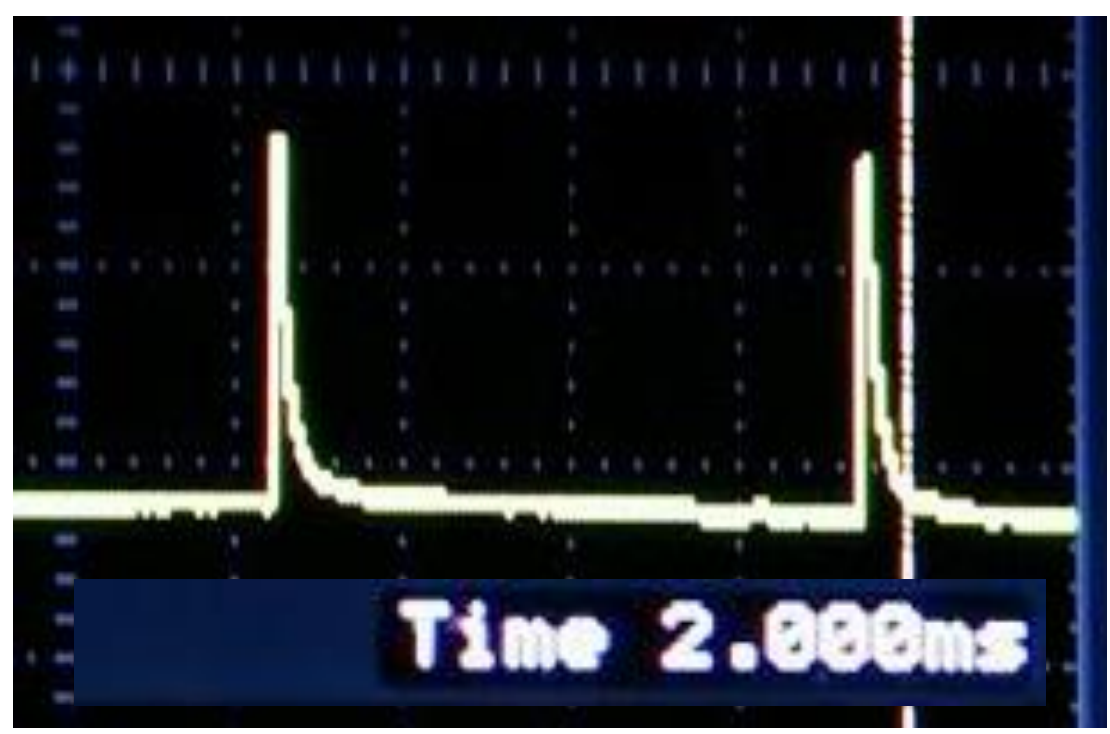

Figure 1: The pulse signals emitted by the automatic door in order to detect the movement. 


\subsection{Infrared Sensor}

An infrared sensor is the device which receives the infrared, converts it to the electric signal, and decodes the information from it. Using infrared, the device can see the objects without affecting person. There are about two kinds of infrared sensors: thermal infrared sensor and quantum infrared sensor.

A thermal infrared sensor receives infrared rays and heats the sensor to detect changes in electrical properties caused by an increase in element temperature. When the temperature difference between the background and the heat source is small, it is difficult to distinguish between the background and the heat source, and when there is no temperature difference, nothing can be detected in principle. It is mainly used for far-infrared sensors that capture farinfrared rays, which are hot electromagnetic waves.

A quantum infrared sensor detects an electric phenomenon caused by light energy. Photodiodes, phototransistors, photo ICs, etc. that are sensitive to the infrared region and have a narrow bandgap are used. It has high detection sensitivity, excellent response speed, and has a detection capacity of 100 to 1000 times that of the thermal type. Sensitivity depends not only on the type of semiconductor used, but also on the wavelength of infrared rays. However, on the other hand, in principle, it is easily affected by thermal noise and detects the heat generated by the image sensor itself, so it is necessary to keep the image sensor at a sufficiently low temperature as compared with the subject. It is mainly used for near-infrared sensors that irradiate near-infrared rays and detect objects by the amount of change due to reflection or transmission.

The infrared sensor used to detect person in automatic doors is a near-infrared sensor that uses a quantum type infrared sensor. Near infrared rays are emitted from the light projecting element mounted on the sensor body, and this light is projected onto the floor surface through the optical system. This projection range is configured as a three-dimensional detection area, and when a person or object enters this area, the amount of reflected light of near infrared rays' changes. The sensor converts the change into a detection signal. In Japan, where automatic doors are currently being used, most of the automatic door activation sensors are near-infrared reflection sensors.

\section{Proposed Detection Method}

Most of automatic doors in Japan use near-infrared reflection sensors. That means, most of them always emit the infrared rays. We can announce the existence of automatic door to the visually impaired person by detecting this infrared rays. Then, the accidents at the automatic door will be reduced.

At first, we discuss where to set the infrared sensor. For example, we put it on the body. The automatic door detects the foot at first because the sensing range is from top to diagonally downward. Then the automatic door already detects the person when the sensor on the body detects the infrared. That means, at that time when the sensor on the body detects the infrared, the door is already opened. And if the person is walking along the wall, she/he are hit by the door. Even if she/he are walking not along the wall, at that time, the distance to door is very close. And it is difficult to avoid the door. Now we focus the white cane which is handled always for moving by the visually impaired person [6].

Since the user always holds the white cane diagonally forward and downward, it enters the detection range of the automatic door before the human body. Furthermore, by attaching an infrared sensor to the tip of the white cane, the infrared rays of the automatic door can be detected 
first (shown in Figure 2). Then, by setting a vibration motor to the grip of the white cane, it is possible to notify the user of the existence of the automatic door by tactile sensation.

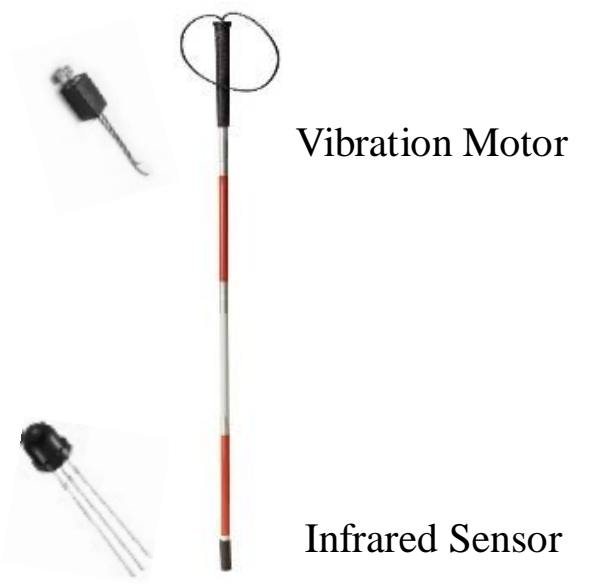

Figure 2: The proposed device with the vibration motor and the infrared sensor which detects the automatic door and tell its existence to the user.

\section{THE EXPERIMENT AND ITS RESULTS}

In order to show the performance of proposed method, we made the prototype system and whether it is safe or not to through the automatic door with it.

The prototype system is shown at Figure 2. The infrared sensor is put at the edge of the white cane. And we use ATmega328P as the microcomputer [7] in order to control the vibration motor. If it detects the pulse wave from the infrared sensor, the vibration motor will start moving. Then the examinee can sense the automatic door sensing area with swinging this cane.

The examinee blindfolds and puts earplugs in order not to know the location by eyes and sound (shown in Figure 3). The examinees start at the 16[m] front of the door. And they try to through the automatic door using proposed white cane. We measure the time when the examinee through the door. All the examinees do not use the white cane usually, and they are not fitting for using the white cane and walking without their senses. We have ten tries which they walk with the conventional white cane and with our proposed white cane.

Figure 4 shows the performance which means the through time with and without our proposed device. When the examinee uses the conventional white cane, which does not have the sensor and the motor, if the door is found quick, it is easy to through the door, and if the door is not found, it requires a lot of time. In particular, if they lost their route to the door, they must find the door using the white cane. That means they must touch the door or the wall. It requires a lot of time. On the other hand, when the examinees use our proposed device, the distribution of time is smaller than that of the conventional method. Once the examinees find the door with the vibration from the white cane, after that they can walk smoothly because they understand the location of the door. That means, this device provides the user reassurance and safety.

This time our proposed system uses one kind of vibration pattern. So, the examinees can get only one information. If we use various kinds of vibration patterns, the system can tell the examinees a lot of information. 


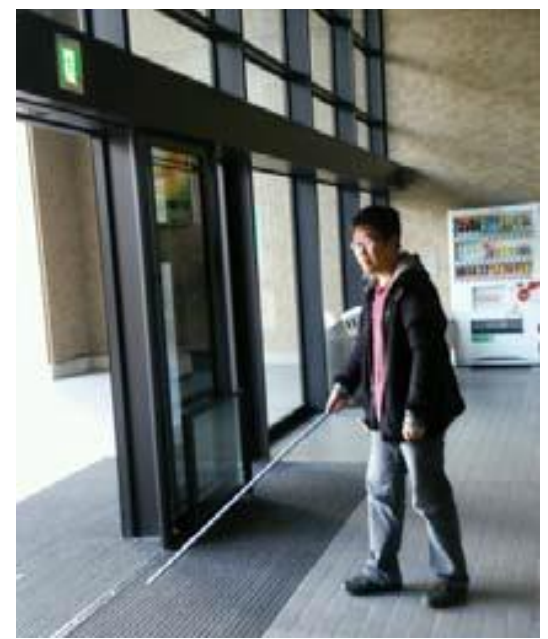

Figure 3: The examinee walks into the automatic door using the proposed white cane without his visible and hearing.

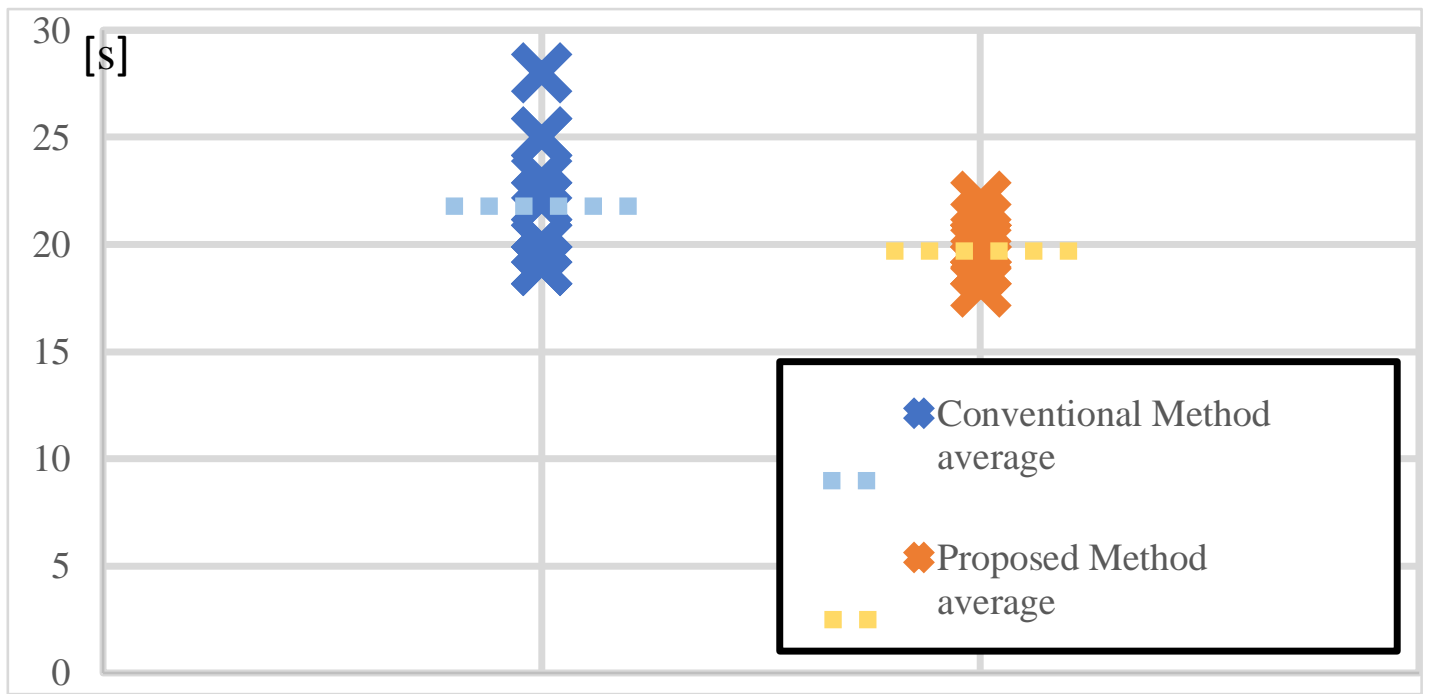

Figure 4: The experimental result shows the through time with and without our proposed device.

\section{CONClusion}

For visually impaired person we proposed the detection method for the automatic doors. By attaching an infrared sensor to the tip of the white cane, the infrared rays of the automatic door can be detected first. And by setting a vibration motor to the grip of the white cane, it is possible to notify the user of the existence of the automatic door by tactile sensation. As a result, the white cane equipped with an infrared sensor reduced the stress when the visually impaired person through the automatic door. For the future work, we would like to update the number of motors. Using the property of moving in the direction of strong vibration, the navigation system will tell the direction to the user, for example, which direction the infrared source is located by the strength of vibration of the motor and its position. And this time, the experimental environment is only one that means we use only one automatic door. So, we will have the experiment for the other automatic doors. For examples, the door which width is different, the door which height is different, the door which pulse wave of infrared is different, and the door which sensor is different from infrared. 


\section{REFERENCES}

[1] Keisuke Hongo, Shinobu Yamazawa, Go Yanagisawa, and Minami Adegawa, "5317 Research of a sensor on automatic doors at stations for Accessibility (1)", Architectural Institute of Japan, Summaries of technical papers of annual meeting, pp. 667-668, (2011).

[2] Minami Adegawa, Shinobu Yamazawa, Go Yanagisawa, and Keisuke Hongo, "5318 Research of a sensor on automatic doors at stations for Accessibility (2)", Architectural Institute of Japan, Summaries of technical papers of annual meeting, pp. 669-670, (2011).

[3] Keisuke Hongo, Takahiro Sugiyama, Go Yanagisawa, and Minami Adegawa, "5450 Research of a sensor on automatic doors at stations for Accessibility (3)", Architectural Institute of Japan, Summaries of technical papers of annual meeting, pp. 941-942, (2012).

[4] Junichi Fujisaku, Rumiko Koda and Katsuharu Nakazato, "Research on Psychological Difficulties on Using a White Cane by People with Visual Impairment", Bulletin of Tokyo University and Graduate School of Social Welfare, Vol4. No. 2, pp. 115-124, 2014-03-25.

[5] Atsushi Kondo, Shunichi Yonemura, "Analysis of direction perception in the sound interface of the electronic game for visually impaired people", IEICE technical report, Vol. 114, No. 357, WIT 2014-56, pp. 17-21, (2014).

[6] Wataru Uemura and Takato Hayama, "A white cane mounted with infrared sensors to detect automatic doors," 2015 IEEE 5th International Conference on Consumer Electronics - Berlin (ICCEBerlin), Berlin, 2015, pp. 54-56, doi: 10.1109/ICCE-Berlin.2015.7391330.

[7] Kashimoto Hiroshi and Hirakoso Nobuto, "Introductory Education of the Microcomputer Control using Arduino", The Gunma-Kohsen review, Vol. 33, pp. 69-77, 2014.

\section{AuTHORS}

Wataru Uemura was born in 1977, and received B.E, M.E. and D.E. degrees from Osaka City University, in 2000, 2002, and 2005. He is an associate professor of the Department of Electronics and Informatics, Faculty of Engineering Science, Ryukoku University in Shiga, Japan. He is a member of IEEE, RoboCup and others.

Takato Hayama was born in 1992 and received B.E, and M.E from Ryukoku University, in 2015, 2017. He is interested in the card games.
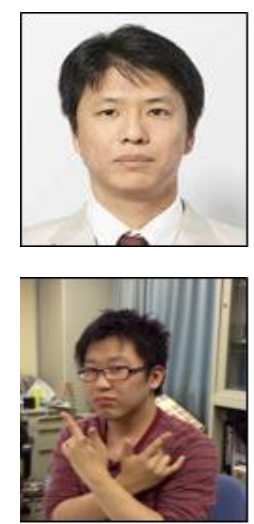